\title{
Cloud Education Survey
}

\author{
Kefei Cheng ${ }^{1, a}$ and Dan Xiao ${ }^{2, b}$ \\ ${ }^{1,2}$ College of computer science and technology, Chongqing university of posts and \\ telecommunications, Chongqing, China \\ achengkf@cqupt.edu.cn, b1007671530@qq.com
}

\section{Keywords: Cloud Computing, Cloud Education, Personalized Recommendation}

\begin{abstract}
As a new computing model and service model, currently, cloud computing has been attracting attention from around the global. As an important means of education reform, cloud education, which means offering a variety of educational services by using advantages of cloud computing, also has drawing concerned from all around the world in the educational field. The paper analyzed the educational inequity actually existing in traditional education caused by the difference of areas, population and other aspects. Then it described some related concepts of cloud education, and put forward a technology solution to solve the problems of hard to share educational resources and unfairness in the cloud computing environment, as well as new issues faced in cloud education. Finally, the article summarized the existing research results in cloud educational applications and proposed the research trends of cloud education in the "Internet +" era.
\end{abstract}

\section{Introduction}

In China, there are wide area and large population, so the development of economic is very uneven in different cities and areas. As a result, education resources, infrastructure and cultural resources are extremely unequal [1]. Based on the above issues, information technology programs of education resources were proposed. However, it is common that schools contact with each other little, and it's serious that repeated construction, investment and "information island", so that the advantages of optimal allocation and sharing cannot be reflected. Additionally, requirements on information and services put forward by users are increasing, so the Internet has to provide more and more services, and these services must handle plenty of information, consequently, the Internet system load has been increasing. How to meet demands on services and massive information, how to make the investment costs be more effective and how to achieve cost-effective sharing mechanism, these problems have attracted researchers' attention.

Fortunately, the emergence of cloud computing provides a new way of thinking to integrate all idle network resources and promote sharing of education resources. Based on cloud education platform, various education information resources can be stored on the "cloud" [2]. On one hand, it provides relevant users complete online learning environment and more convenient learning tools. Therefore, users are able to study as they will, that is to say, they can freely choose the time, address and content to study. On the other hand, when users select the tools, utilize the resources and communicate with others to solve their problems, they do these by their willing and form their own experience and cognitive structure. In sum, cloud education will promote sharing of education information resources, facilitate communication between teachers and students and strengthen cooperation between schools and schools, between schools and employers. At the same time, it will have a vital impact on maximizing the use of all kinds of education resources.

\section{Related Concepts of Cloud Education}

\section{Cloud education}

As we all know, cloud computing has benefits such as very large scale, high reliability and high scalability. Cloud education means offering a variety of educational services on the cloud platforms based on using the advantages. It breaks the boundaries of traditional education information and introduces the new concept of education information. Same time, it gets teaching, management, 
learning, entertainment, sharing and interaction together. So persons, such as parents, education sectors, with different identities can finish different work on the same platform, according to their own rights. By taking advantage of cloud computing technology model, it realizes distribution management and sharing of teaching resources and seamless integration of teaching resources and teaching [3], which meet the demand of teachers' innovative teaching, students' personal learning and education managers' efficient management. Essentially, cloud education changes the way of education and is the external performance of education information and networking.

\section{Key technologies of cloud education}

(1)Cloud computing technology.

Cloud computing is a new computing model and service, which is the converged product of traditional computers and network technologies including grid computing, distributed computing, parallel computing, virtualization, load balancing, network storage and so on [4]. It distributes complex computing tasks on resource pool which is made of a large number of computers, so various application systems can obtain computing power, storage space and a variety of software services based on their own needs.

(2)Storage and management technologies of distributed massive data.

Cloud computing system consists of a number of servers using distributed storage mode for storing data, Cloud computing system consists of a number of servers using distributed storage mode for storing data, and it uses this method of redundant storage to ensure the data's reliability. As to redundant storage, it lowers the cost by the tasks' decomposition and clustering and applying poor machines to substitute super computers. Nowadays, data management technologies mainly have BT sT IO from Google and open source data management module HBase developed by Hadoop [5].

(3)Personalized recommendation algorithms.

Personalized recommendation algorithm of education resources means that recommending teachers and students resources which they are interested in most, based on the users' own interests, requirements and periodic characteristics of studying. Under the background of massive data mining, personalized recommendation system utilizes effective algorithms of clustering and association rules, aiming to help users make decisions and provide advanced, intelligent and personalized platform.

\section{Advantages of cloud education}

Cloud education is very powerful and has kinds of advantages.

(1) As cloud education integrates cloud computing, cloud storage and intelligent terminal technology, so it can effectively solve the problem of sharing hardware and software resources difficultly through personal computers or smart terminals.

(2) It realizes the combination of traditional web models based on browsers and intelligent terminals. As a result, it both offers users traditional using methods and new learning models and greatly improves the teaching efficiency.

(3) On the one hand, in remote underdeveloped areas with lack of teaching resources and outdated, cloud education can access to the network of unified teaching resources cloud quickly and easily. On the other hand, the security issues of data in transmission and users' information can be guaranteed by limiting the management rights.

(4) Cloud education has many other merits, such as cost less, application richer, unlimited storage space and access easier.

\section{Design of Cloud Education Platform}

\section{Architecture of cloud education platform}

The architecture of cloud education consists of 4 layers, including infrastructure layer, application interface layer, application layer and users interface layer. As shown in Figure 1. 


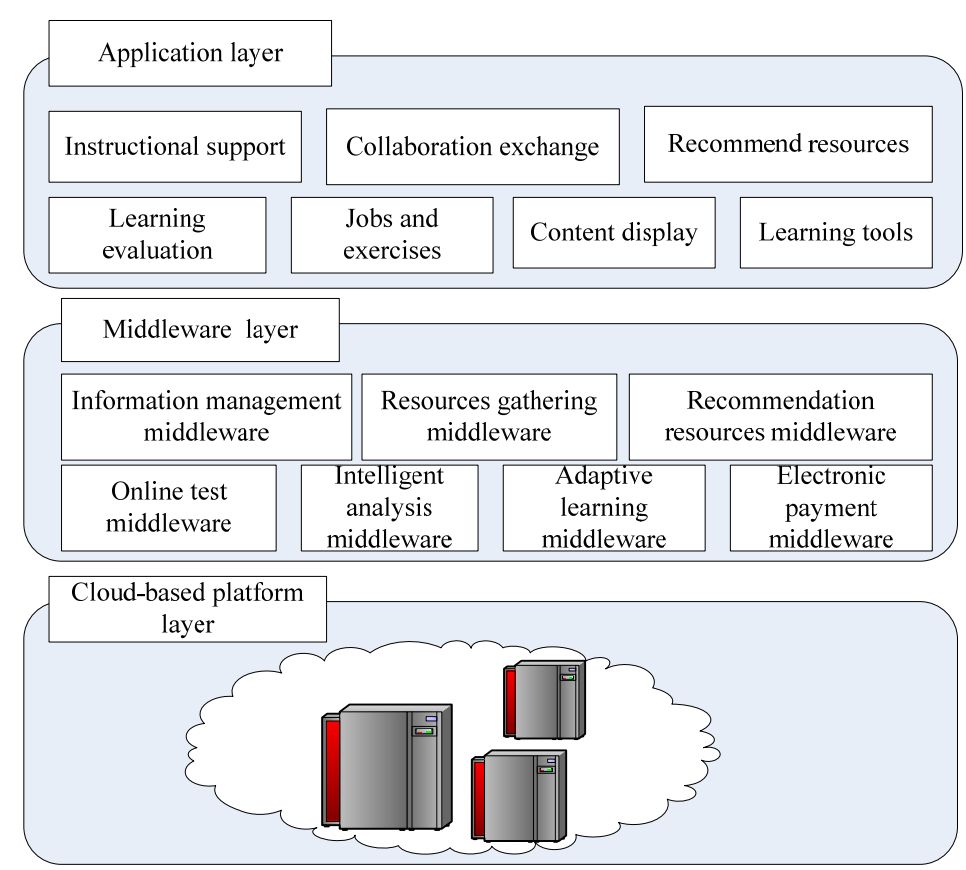

Figure 1 Architecture of cloud education

a) Cloud-based platform layer: the layer providers cloud education the cloud platform. The platform provides storage, network and computing power and so on to support the upper layer, so the services on the upper layer will carry out more smoothly than common platforms.

b) Middleware layer: it offers lots of services platforms. Information management middleware manages users' usually records, verification and registration. Resources gathering middleware collects all learning resources and basic information. Recommendation resources middleware is to recommend resources and answers according to users' habits and existing resources. Online test middleware offers students examination papers to test their mastery of knowledge. Intelligent analysis middleware analyzes users' information and obtains models to match existing resources. Adaptive learning middleware provides users learning resources which comply with their own habits. Users need to pay come money to enjoy shared learning resources and electronic payment middleware checks the pay and date.

c) Application layer: the layer has kinds of services. Instructional support module supports teachers publish course announcements, upload teaching resources, organize teaching activities, correct students' homework and arrange group collaboration. Content display module adaptively shows two-dimensional and three-dimensional learning resources on different terminals including personal computers, tablet PCs, smart phones and network TV. Recommend resources module establishes students' models, captures learners' characteristics and personal interests, and it recommends different types of learning resources to learners to meet the needs of different learners. Jobs and exercises module supports learners complete the courses' work and related exercises by using different intelligent terminals. Collaboration exchange module establishes learning social networks and learning community, meanwhile, it supports collaboration and communication between learners and learners, learners and teachers. Learning evaluation module tracks, analyzes and monitors learners' learning process, providing learning recommendations, and evaluates learning outcomes to provide a basis for improving teaching. Learning tool module provides a variety of learning tools for learners, facilitating record their learning content and track the process and so on.

\section{Implementation scheme of cloud education}

The paper adopts B/S (browser/server) model and offers traditional GUI and B/S architecture on the web. Based on that, the cloud education offers instructional support module, content display module, recommend resources module, jobs and exercises module, collaboration exchange module, learning evaluation module and learning tool module to help users study better.

(1) Basic learning. 
After verified users' information successfully through the information management middleware, users $\log$ in the web. As to teachers, for one thing, they can correct students' homework, arrange group collaboration and upload their own resources which will be gathered with others by resources gathering middleware. For another, they will be recommended many resources based on their habits and interests through recommendation resources middleware. As to students, they can test online, download resources and so on by these middleware. At the same time, the cloud platform will store users' records.

(2) Personalized learning.

It's known that everyone has its own advantages and disadvantages and habits of studying. To improve users' learning efficiency, the paper introduces personalized learning mechanisms through the recommendation resources middleware. The mechanisms tailor users' learning plans according to their existing knowledge and abilities of learning. First, according to the user registration information and habits of browsing webs, collect users' basic information and preference. And then analyze various properties of learners and learning resources on the resources gathering middleware, obtain the association between learners and learning resources by intelligent analysis middleware. After then, applications make combined analysis between previous results and learning resources' properties regularly by intelligent analysis middleware and adaptive learning middleware. Then, frontend application passes in operating properties run in the current by users, recommendation engine online merger, analysis, filtering them and properties from databases to achieve personalized recommendation of learning resources. Taking into account that users' information is dynamically changing, traditional personalized recommendation algorithms on learning resources are difficult to meet the application contexts, thus the paper integrates collaborative filtering recommendation algorithm and based on content recommendation algorithm. Meanwhile, it designs an approximate dynamic algorithm to solve data information's change by topically changing the original results and introducing algorithms of recommendation based on demographic information and Slope One Filling.

(3) Community learning.

In order to help solve users' problems at any time, learning communities are established. First of all, according to users' profile and interest, it analyzes users' level of knowledge, cognitive ability, learning motivation, learning style, changing rules of learning habits and so on through intelligent analysis middleware and information gathering middleware. After then, build users' learning models based on the characteristics with adding timeliness. Next, divide users into learning communities based on similarity, so users can discuss their questions in the communities. At the same time, it adopts graph-based recommendation algorithms [6] and the language processing model of LDA (Latent Dirichlet Allocation), so the system will recommend questions to others who have a high probability to answer the questions correctly. Meanwhile, users who put forward questions will also receive some questions suggested by recommendation resources middleware. As a result, the degree of discussion is improved and the questions will be solved quicker and more accurate.

\section{The Existing Problems in the Development of Cloud Education}

Since 2008, cloud computing was introduced into China and has been developing rapidly, even became a hot topic in educational technologies. Recent years, cloud computing's application in the field of education has been favored by researchers. However, the application is still in its infancy with many speculative qualitative researches and lack of empirical and quantitative researches. In the meantime, there are not enough in-depth researches. Specific problems are included as follows.

\section{Construction standards of cloud computing are not yet developed in advance.}

Most R\&D (research and development) institutions are built by their own and adopt different hardware, software and programming languages. Since there are no uniform standards of cloud education, it's hard to achieve effective sharing among institutions and leads to phenomena of "islands", "isolated cloud" and so on [7]. On the one hand, at present, formats' kinds about digital learning content are as many as 30, including EPUB, PDF, CWBX, TXT, but there is not a unified format. Moreover, standardization learning resources' content formats is the prerequisite and basis to 
improve services and manage effectively, which will avoid incompatibilities happened in re-development, exchange and sharing effectively. On the other hand, there are a number of packaging technologies standards on learning content, such as IMS learning resources standards, SCORM and domestic CELTS. Hence, the standards still need further study and norms.

\section{Researches need to be thorough.}

Nowadays, that cloud computing applications in education is widely accepted by all of us, but most of the researches on cloud education' applications are still in their infancy and relatively lack of development researches of innovative applications of cloud computing. Simultaneously, many related technologies are still in development, such as virtualization technology, effectively management of plentiful data, intensive computing's effective scheduling, security assurance of data and computing resources. It specifically includes as follows.

a) Ensure the effective operation of education business by computing services and data storage services' availability provided by cloud computing mode.

b) Preventive technologies of security to avoid loss and destruction of cloud storage data, so the related data is protected.

c) Solve the problem of massive data's transmission and achieve using data transparently.

d) Take advantage of virtualization technology to deal with rapidly changing of customers' needs and service level agreement cost-effectively and telescopically.

\section{Application Summary and Development Trend of Cloud Education}

\section{Researches summary of cloud education applications}

Recent years, education application researches have begun to focus on cloud computing applied in the construction of primary and secondary education. Nanhai District of Foshan City in Guangdong Province has set up education cloud, focusing on exploring "ubiquitous learning" system [8]. It aims to build intelligent education information network service system based on cloud. After then, the Ministry of Education introduced policies to support the development of cloud computing in education in China and the "China Academic Conference Online" used the cloud technologies which was hosted by Ministry of Education's Education and Technology Development Center. To promote teaching on the network projects, the Ministry of Education Curriculum Materials Center took "Zhi Hao Le education cloud" as a public service platform built by Tong Fang. It focused on the construction of primary and secondary education. In 2011, the Ministry of Education and various educational authorities in various regions surely treat building cloud education as the focus of the current and near-term educational construction work. So far, various educational clouds have sprung up in China, for example, Asian Education Network, National Education cloud, Wuhan Public Service Platform and so on.

\section{Cloud education research tendency in the "Internet+" era}

According to a research report from the Zero2IPO Group's "the Private Tong", online education financing has nearly occurred 100 cases from early 2015 to date, totaling nearly $\$ 1$ billion [9]. Currently, mobile learning, ubiquitous learning and lifelong learning have become hot issues in educational technologies in China. Considering the space-time dimension, cloud education's tendency is mobile learning. Thinking of access to resources, it tends to select ubiquitous learning. As to the common aspiration, it is willing to choose the lifelong learning. Therefore, improving theories of cloud education is very crucial and it's a necessary condition of practicing of mobile learning, ubiquitous learning and lifelong learning. In addition, effective use of multiple learning styles can not only enrich teaching methods and approaches, but also provide personalized development space to users.

\section{Summary}

Cloud education has many incomparable advantages on resources sharing comparing to traditional education, and brings an entirely new idea and method. It upgrades education information level between regions, urban and rural areas, schools. Despite there are lots of bottleneck, cloud education 
will still be the construction infrastructure of education information in future, and it cracks island effect and reduces resources gap among different schools. In the form of public cloud resources, abundant high-quality education resources on cloud will offer free for underdeveloped remote rural between regions, which will contribute to educational equity, higher quality of education and reducing the waste of resources.

\section{References}

[1] Zhang X, Ma H, Wu Y, et al. Applying cloud computing technologies to upgrade the resource configuration of laboratory course: The case of quality engineering education platform[J]. International Journal of Engineering Education, 2014, 30(3): 596-602.

[2] Lin H, Wang Y, Cai J, et al. Design and development of education cloud platform[J]. Journal of Chemical \& Pharmaceutical Research, 2014, 6(3).

[3] Grover J, Kheterpal G. Mobile Cloud Computing: An Introduction[J]. Resource Management of Mobile Cloud Computing Networks and Environments, 2015: 1.

[4] González-Martínez J A, Bote-Lorenzo M L, Gómez-Sánchez E, et al. Cloud computing and education: A state-of-the-art survey[J]. Computers \& Education, 2015, 80: 132-151.

[5] Bai W, Geng W. Research on Operation Management under the Environment of Cloud Computing Data Center[J]. International Journal of Database Theory \& Application, 2015, 8(2).

[6] Xu B, Yang D. Study Partners Recommendation for xMOOCs Learners[J]. Computational intelligence and neuroscience, 2015, 2015.

[7] Grover J, Sharma M. Cloud computing and its security issues - A review[C]//Computing, Communication and Networking Technologies (ICCCNT), 2014 International Conference on. IEEE, 2014: 1-5.

[8] http://www.jszpw.net/news/news-show.php?id=1406.

[9] http://snap.windin.com/ns/findsnap.php?id=240724615. 